\title{
Attentional requirements in perceptual grouping depend on the processes involved in the organization
}

\author{
Einat Rashal $^{1} \cdot$ Yaffa Yeshurun $^{1} \cdot$ Ruth Kimchi $^{1}$
}

Published online: 5 July 2017

(C) The Psychonomic Society, Inc. 2017

\begin{abstract}
Previous studies on the role of attention in perceptual grouping have yielded contradicting findings, some suggesting that grouping requires attention and others indicating that it does not. Kimchi and Razpurker-Apfeld (Psychonomic Bulletin and Review, 11(4), 687-696, 2004) showed that attentional demands in grouping could vary according to the processes involved. The current study expanded on this, examining whether attentional demands vary for (a) different grouping principles and (b) as a function of contingent processing of element segregation and shape formation. We used the inattention paradigm with an online measure, in which participants engaged in an attentionally demanding changedetection task on a small matrix presented on a taskirrelevant backdrop of grouped elements. The backdrop grouping changed or stayed the same independently of any change in the target. Congruency effects produced by changes in backdrop grouping on target-change judgments indicate that the backdrop grouping was accomplished under inattention. The results showed congruency effects when grouping formed columns/rows by proximity but not by shape similarity, and when grouping into a distinct shape by collinearity did not involve element segregation. No congruency effects were found when grouping into a shape by collinearity or connectedness involved element segregation, except when connectedness was combined with color similarity. These results suggest that attentional demands depend on the combination of grouping principles and the complexity of the processes involved in the organization. These findings provide further support for
\end{abstract}

Einat Rashal

einatrashal@gmail.com

1 Department of Psychology and Institute of Information Processing and Decision Making, University of Haifa, Haifa 3498838, Israel the view that perceptual organization is a multiplicity of processes that vary in attentional demands.

Keywords Attention - Divided attention and inattention . Grouping and segmentation · Perceptual organization

When we view our surroundings, a massive amount of unstructured visual information falls on the retina. Processes of grouping and figure-ground segmentation aid the construction of the bits and pieces of this information into a coherent percept of the environment. The traditional theories of perceptual organization assumed that grouping and figure-ground segmentation are early, low-level, preattentive processes that provide preliminary units for more elaborate attentional processing (e.g., Julesz, 1981; Marr, 1982; Neisser, 1967; Treisman, 1982). Today, evidence accumulates in favor of a multistage, iterative process that operates on visual representations before and after constancies and depth are achieved (Palmer, Brooks, \& Nelson, 2003). Moreover, perceptual organization is now thought of as a multiplicity of processes, instead of a single, unified process (Behrmann \& Kimchi, 2003; Kimchi, 2003; Kimchi \& Razpurker-Apfeld, 2004). This view is supported by findings from recent studies demonstrating that different grouping organizations have different time courses, developmental trajectories, and attentional demands (e.g., Behrmann \& Kimchi, 2003; Ben-Av \& Sagi, 1995; Ben-Av, Sagi, \& Braun, 1992; Hadad \& Kimchi, 2006; Kimchi, 1998, 2000; Kimchi, Hadad, Behrmann, \& Palmer, 2005; Kimchi \& Razpurker-Apfeld, 2004; Kurylo, 1997; Quinn, Bhatt, Brush, Grimes, \& Sharpnack, 2002).

Attentional demands in grouping have been investigated in the past decades and yielded inconsistent results (e.g., Ben-Av et al., 1992; Kimchi \& Razpurker-Apfeld, 2004; Lamy, Segal, \& Ruderman, 2006; Mack, Tang, Tuma, Kahn, \& Rock, 1992; 
Moore \& Egeth, 1997; see Kimchi, 2009, for a review). For example, Mack et al. (1992) showed that when observers were engaged in an attentionally demanding task, they could not report grouping organizations that were presented in the unattended backdrop of the task-relevant stimulus. Thus, they concluded that the organizations could not be perceived without attention. In the same line, Ben-Av et al. (1992) showed that identification of grouping organizations was severely impaired when observers were asked to report the organization in a secondary task, compared with when it was their primary task. In contrast, several studies have provided evidence that some grouping organizations can be perceived without attention when indirect, online measures were used instead of explicit reports of the observers' perception of the organization (Driver, Davis, Russell, Turatto, \& Freeman, 2001; Kimchi \& Razpurker-Apfeld, 2004; Lamy et al., 2006; Moore \& Egeth, 1997; Russell \& Driver, 2005). For example, Moore and Egeth (1997) devised a paradigm in which participants were asked to judge which of two lines is longer. The lines were embedded in a display composed of a large number of elements in two different colors. On the critical trials, the elements of the display were grouped by color similarity, forming an organization that subjected the lines to the influence of the Müller-Lyer or Ponzo illusions. They reasoned that if attention is unnecessary for grouping to occur, the illusions should influence the observers' performance even when they were not attending to the grouping display. Indeed, they found that the line discrimination task was affected by the illusions, although the observers could not report what was presented in the backdrop. Thus, Moore and Egeth (1997) provided evidence for grouping without attention when using an indirect online measure that did not rely on the memory of the organization.

Later, Kimchi and Razpurker-Apfeld (2004) showed that grouping under inattention is not an all-or-none effect, but it depends on the processes involved in the organization. In their study, they used an inattention paradigm first described in Driver et al. (2001; see also Russell \& Driver, 2005). In that study, participants engaged in an attentionally demanding change-detection task on a small matrix of black and white pixels that was presented briefly at the center of the screen. On each trial, the observers were presented with two consecutive displays and were asked to indicate whether the matrices in the two displays were the same as each other or different. Importantly, the central matrix appeared on a task-irrelevant backdrop of elements that were grouped by color similarity. The organizations in the backdrop changed or stayed the same for the two consecutive displays independently of whether the central matrix stayed the same or changed. This design allowed assessing whether a change or no change in the backdrop grouping affects the change judgments for the central matrix, thus providing an online, indirect measure for the processing of the unattended organizations. Their results showed that grouping organizations of columns/rows by color similarity did not require attention, and the same was true when a single group of elements of the same color was grouped into a distinctive shape (e.g., square or cross). However, attention was required when one group of elements formed a shape or a line, while surrounded by elements of a different color. The authors suggested that in these cases attention was required in order to resolve figure-ground relations between the groups segregated by color and concluded that the demand for attention depends on the processes involved in the organization.

Two important unresolved issues concerning the role of attention in grouping emerge from Kimchi and RazpurkerApfeld's (2004) study. First, since the only grouping principle examined in that study was color similarity, attentional demands for other grouping principles remain unclear. Second, the suggestion that attention is required to resolve figureground relations is somewhat weakened by the finding that figure-ground segmentation could occur under inattention when it was based on convexity (Kimchi \& Peterson, 2008). Therefore, it is possible that attentional demands are not necessarily related to a competition for figural assignment but rather to the mere involvement of contingent processes of element segregation and shape formation in achieving the grouping organizations. Element segregation refers to the process of determining which elements belong together and are separated from other elements, and shape formation refers to the process that determines how the grouped elements appear as a whole based on the interrelations of the elements (Koffka, 1935; Rock, 1986; Trick \& Enns, 1997).

To address these issues, we first examined whether attentional demands vary for different grouping principles, by comparing the demand for attention in grouping elements into columns/rows by proximity (Experiment 1 ) and by shape similarity (Experiment 2). Using the same grouping organization of columns/rows, we could assess the differences between the underlying grouping principles. Next we investigated attentional demands for grouping organizations that involved contingent processes of element segregation and the formation of a distinctive shape (square/cross by collinearity, or square/ triangle by element connectedness) with no (or almost no) need for figure-ground relations to be resolved (Experiments 3, 5, and 6). For comparison, we examined attentional demands for grouping organizations in which shape formation (same shapes as in Experiment 3) is not contingent on element segregation (Experiment 4).

In all the experiments reported here, the inattention paradigm with an online measure was used to determine whether or not the organization under study could be accomplished without attention (Driver et al., 2001; Kimchi \& Peterson, 2008; Kimchi \& Razpurker-Apfeld, 2004; Russell \& Driver, 2005). A trial consisted of two consecutive displays, each of which included a small matrix comprised of randomly arranged black and white pixels, presented on the center of a 
task-irrelevant backdrop depicting a grouping organization. The participants' task was to judge whether or not a change had occurred in the matrix between the two displays. On same-response trials, the matrix was identical in the two displays, and on different-response trials, it differed in a location of one of the white pixels, rendering the task sufficiently demanding to absorb attention and leaving the backdrop unattended. The backdrop grouping organization stayed the same or changed across the two displays, independently of whether or not the target matrix changed. We examined whether the backdrop organization influenced performance on the target change-detection task. If the backdrop organization is accomplished, then congruency effects are expected to emerge, indicated by an interaction between the backdrop organization and the target task, such that target-same judgments are expected to be faster and/or more accurate when the backdrop organization stays the same than when it changes, and targetdifferent judgments are expected to be faster and/or more accurate when the organization in the backdrop changes, than when it stays the same. After the last experimental trial, participants were probed with surprise forced-choice questions about the immediately preceding backdrop organization and whether the organization changed between the two displays on that trial.

\section{Experiments 1 and 2}

Grouping principles govern the linking of some elements and not others in the display according to some property (e.g., color, location, shape). Experiments 1 and 2 investigated whether different grouping principles have different attentional requirements.

It was previously reported that grouping elements into columns/rows by color similarity could be achieved under inattention (Driver et al., 2001; Kimchi \& RazpurkerApfeld, 2004; Russell \& Driver, 2005). Kimchi and Razpurker-Apfeld (2004) suggested that attention was not required for columns/rows organization to be achieved presumably because no figure-ground relations needed to be resolved, as all the segregated groups (rows or columns) could be equally designated as figures. If attention is needed only to resolve figure-ground relations, then a columns/rows organization should be achieved without attention regardless of the specific grouping principle underlying the organization. However, if resolving figure-ground relations is not necessary to impose attentional demands, and attentional demands can vary for different grouping processes (Kimchi \& RazpurkerApfeld, 2004), then a columns/rows organization may or may not be achieved without attention depending on the grouping principle underlying the organization. To test this hypothesis, we examined grouping elements into columns/rows by proximity (Experiment 1) and by shape similarity (Experiment 2).
These grouping principles were found to be different in several respects. Proximity is considered a primary and dominant grouping principle that operates at very short durations (BenAv \& Sagi, 1995; Ben-Av et al., 1992; Kurylo, 1997), and it relies on the relative distances of the local elements, which are already available at a very early stage of visual processing (Han, 2004; Han, Song, Ding, Yund, \& Woods, 2001). Grouping by shape similarity, on the other hand, requires more time in order to be detected (Ben-Av \& Sagi, 1995; Ben-Av et al., 1992), and it may rely on shape detection of the individual elements, which was argued to be an effortful process (Rock, Linnett, Grant, \& Mack, 1992). In light of these differences we hypothesized that attention might be required for shape similarity grouping but might not be required for proximity grouping. Specifically, congruency effects produced by changes in the backdrop grouping upon performance on the target are expected to emerge when the organization in the backdrop is constructed by proximity but not when it is constructed by shape similarity.

\section{Method}

\section{Participants}

Twenty students from the University of Haifa participated in Experiment 1, and another 31 participated in Experiment 2. All had normal or corrected-to-normal vision and normal color vision, and all were naïve to the purpose of the study. The Human Ethics Committee of the University of Haifa approved this study, and all the participants provided a written informed consent in accordance with the Declaration of Helsinki.

\section{Apparatus}

The stimuli were presented using E-Prime 2.0 software (Psychology Software Tools, Pittsburgh, PA) on a 22-in. LCD Samsung 2233RZ monitor controlled by a Workstation HP Z400 computer. The experiment was conducted in a dimly lit room. The participants rested their heads on a chinrest at a viewing distance of $57 \mathrm{~cm}$ and watched the screen through a circular aperture of a matte black cardboard sheet.

\section{Stimuli}

Examples of the displays are presented in Fig. 1. Each display consisted of a central target surrounded by backdrop elements. The central target was made of 12 black and 13 white small solid squares, $0.19^{\circ}$ each, randomly located in a $5 \times 5$ matrix subtending $0.95^{\circ} \times 0.95^{\circ}$. The central target matrix stayed the same between two successive displays in half of the trials and changed in the other half. The change was made by switching the location of one small white square with one small black square within the matrix. 


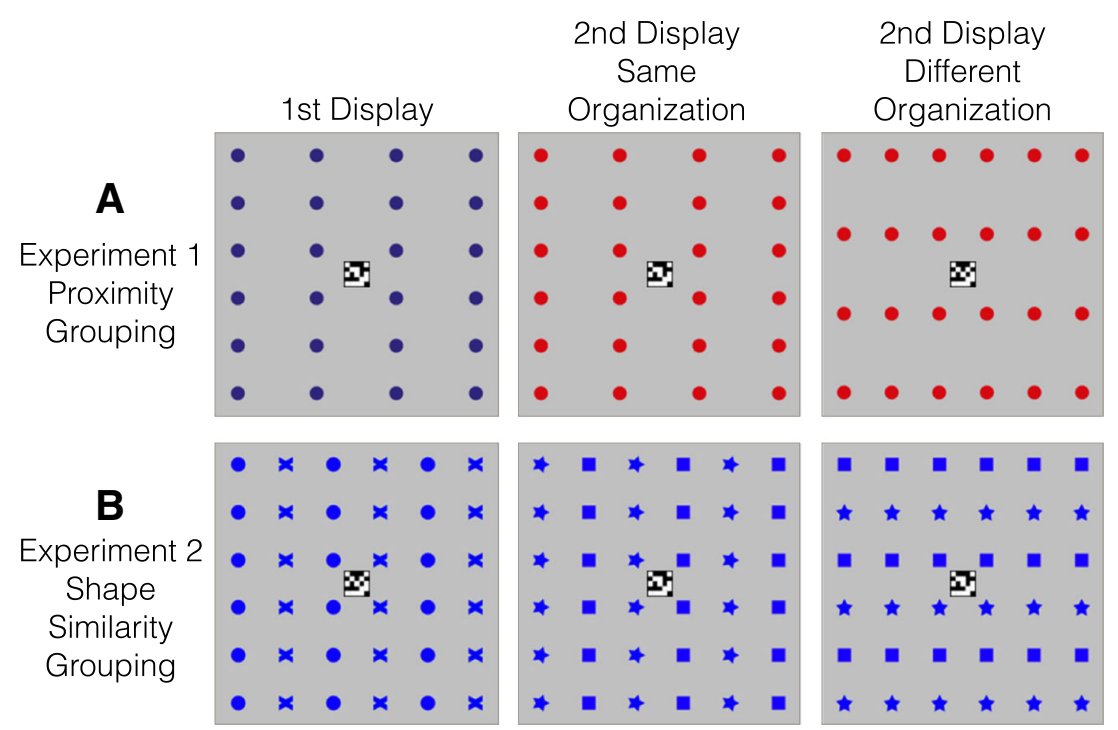

Fig. 1 Example of the backdrop stimuli employed in (a) Experiment 1 (proximity grouping) and (b) Experiment 2 (shape similarity grouping). (Color figure online)

Experiment 1: Proximity grouping Each backdrop display consisted of 24 red or blue solid circles, each $0.48^{\circ}$ in diameter, on a light-gray background, presented in a $9^{\circ}$ $\times 9^{\circ}$ square matrix. The circles were grouped into rows or columns by manipulating the horizontal and vertical proximity between the elements (see Fig. 1a). In the rows display, the horizontal distance between adjacent elements was $1.2^{\circ}$, and the vertical distance was $2.3^{\circ}$, producing four rows of six elements each. In the columns display, the horizontal and vertical distances were reversed, producing four columns of six elements each. The distance of the most central elements from the target matrix was $0.5^{\circ}$. The elements always changed color between successive displays (red in the first and blue in the second, or vice versa), independently of a change in the backdrop organization, to reduce the possibility of detecting backdrop organization changes from local changes in the elements. ${ }^{1}$

Experiment 2: Shape similarity grouping The elements in the backdrop were 36 equidistant, blue solid shapes (circles, $\mathrm{Xs}$, squares, or stars), each subtended $0.5^{\circ} \times 0.5^{\circ}$. The distance between every two shapes was $1.2^{\circ}$, and the distance of the most central shapes from the target matrix was $0.2^{\circ}$. The 36 elements, 18 of one shape (e.g., squares), and 18 of another

\footnotetext{
${ }^{1}$ Given that the backdrop grouping was based on proximity, the ideal way to disentangle a change in the backdrop organization from a local change in the elements is to introduce constant changes in the locations of the elements from the first to the second display; this, however, is very difficult to do without disrupting the grouping of the elements into columns/rows. Therefore, we introduced constant local changes in the color of the elements, which presumably reduced (if not eliminated) the possibility of detecting backdrop organization changes from local changes in the elements.
}

shape (e.g., stars), were grouped into columns or rows by shape similarity: the shapes alternated on the horizontal or vertical axes, respectively (see Fig. 1b). The shapes of the elements always changed between successive displays (circles and Xs in the first, and squares and stars in the second, or vice versa), regardless of whether or not the grouping changed, to control for the possibility of detecting a change in the backdrop grouping from a local change in the shape of just a few elements.

\section{Design}

In each experiment a 2 (target: same, different) $\times 2$ (backdrop organization: same, different) within-subjects design was used. All the combinations of backdrop organization and target were randomized within blocks, with each combination occurring on an equal number of trials. On half of the trials, the target changed between successive displays, and on the other half of the trials, the target stayed the same. Independently of whether the target changed or remained the same on each trial, the organization in the backdrop (i.e., columns/rows) also changed or remained the same. There were 160 experimental trials administered in two blocks, preceded by 16 practice trials.

\section{Procedure}

The sequence of events in a trial is depicted in Fig. 2. Each trial started with a fixation cross that appeared for $250 \mathrm{~ms}$ in the center of the screen. After an interstimulus interval (ISI) of $250 \mathrm{~ms}$, the first display appeared for $200 \mathrm{~ms}$ followed by a $150 \mathrm{~ms}$ ISI, after which the second display appeared for $200 \mathrm{~ms}$. Then, the participants had to indicate whether the two successive central matrices were 


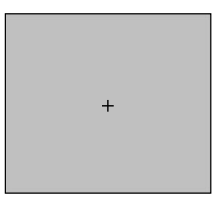

Fixation

$250 \mathrm{~ms}$

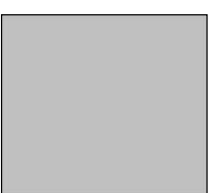

ISI

$250 \mathrm{~ms}$

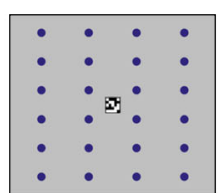

1st Display

$200 \mathrm{~ms}$

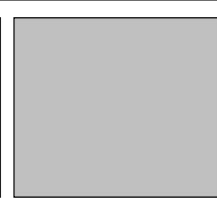

ISI

$50 \mathrm{~ms}$

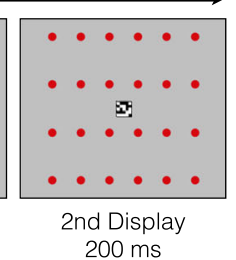

$200 \mathrm{~ms}$

Fig. 2 The sequence of events in a trial. The illustration depicts an incongruent trial, in which the same central matrix (i.e., same target condition) appears on a backdrop that changes from columns to rows (i.e., different backdrop organization condition). (Color figure online)

the same or different by pressing one of two designated keys on the keyboard. The participants were instructed to respond as rapidly and accurately as possible. Reaction time (RT) was measured from the appearance of the second display until a response was made. Feedback about an incorrect response was provided by an auditory tone as soon as the participant responded or if no response was made within 3 seconds. The intertrial interval (ITI) was $1,000 \mathrm{~ms}$. To ensure that the observers were not attending the backdrop displays, they were asked questions about the backdrop display immediately following the last trial (e.g., Kimchi \& Peterson, 2008; Kimchi \& RazpurkerApfeld, 2004). The first question asked was, "What was the pattern in the background?" The two alternatives were columns or rows. The second question asked was, "Was there a change in the pattern in the backdrop organization across the two displays in the previous trial?" The two alternatives were change and no change. In Experiment 2 a third question was asked: "What were the shapes in the backdrop in the last display?" The two alternatives were squares and stars or circles and Xs.

\section{Results and discussion}

All RT summaries and analyses are based on participants' mean RTs for correct responses. Accuracy and RT data were each subjected to repeated-measures two-way analysis of variance (ANOVA; Target $\times$ Backdrop Organization), for each experiment. RTs less than $250 \mathrm{~ms}$ and greater than $2,000 \mathrm{~ms}$ were discarded $(0.5 \%$ and $0.3 \%$ of all trials in Experiments 1 and 2, respectively). Figure 3 depicts mean accuracy rates (ACs) and correct RTs for same and different targets as a function of backdrop organization (same, different), for Experiment 1 (see Fig. 3a; proximity grouping), and Experiment 2 (see Fig. 3b; shape similarity grouping).

\section{Experiment 1: Proximity grouping}

The ANOVA showed a significant main effect of backdrop on accuracy, but not on RT, indicating that responses were more accurate (by 3.3\%) when the organization in the backdrop stayed the same than when it changed: AC, $F(1,19)=$ $10.94, p<.005, \eta_{\mathrm{p}}{ }^{2}=0.37$; RT, $F(1,19)=2.5, p>0.13, \eta_{\mathrm{p}}^{2}$
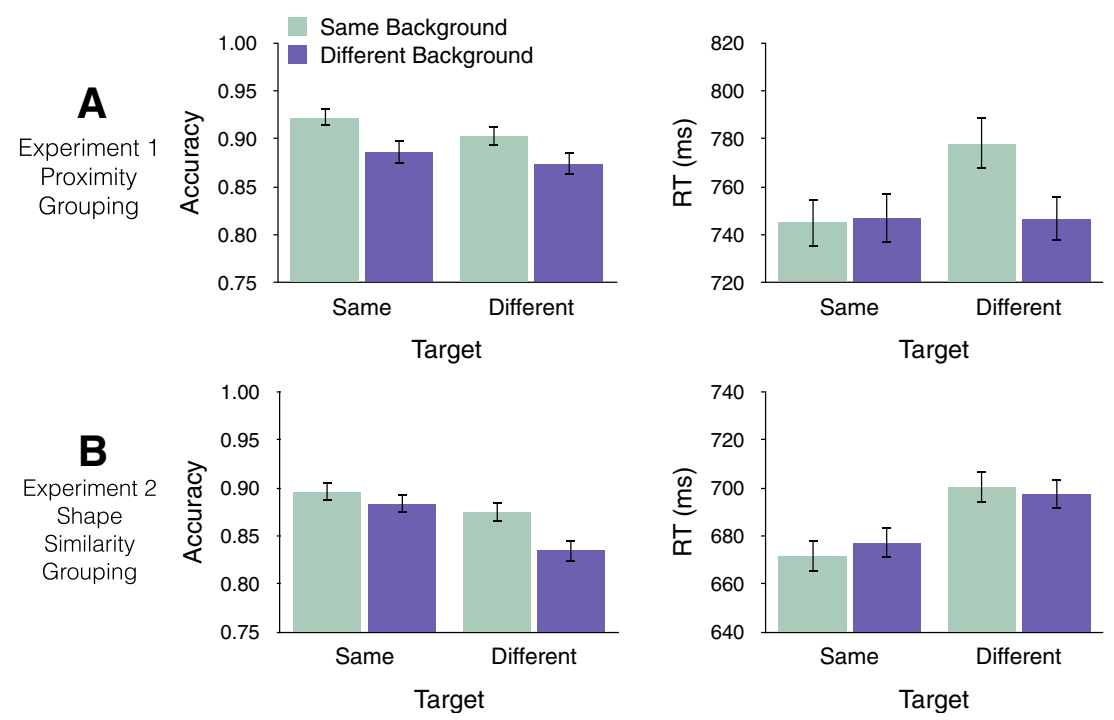

Fig. 3 Mean accuracy (left column) and correct RTs (right column) for same and different targets as a function of backdrop organization (same, different) in (a) Experiment 1 (proximity grouping) and (b) Experiment 2

(shape similarity grouping). Error bars indicate standard errors of the means. (Color figure online) 
$=0.12$. The effect of target did not reach significance for the accuracy data $(F<1)$ and was marginally significant for RT, indicating that same responses tended to be faster (by $17 \mathrm{~ms}$ ) than different responses, $F(1,19)=3.15, p=0.09, \eta_{\mathrm{p}}{ }^{2}=0.14$. Critically, there was a significant interaction between target and backdrop organization for the RT data, $F(1,19)=7.92$, $p<.02, \eta_{\mathrm{p}}^{2}=0.29$, suggesting some congruency effect. No significant interaction was observed for accuracy $(F<1)$. Planned comparisons showed that target-different judgments were faster (by $25 \mathrm{~ms}$ ) when the backdrop grouping changed than when it stayed the same, $F(1,19)=6.6, p<.02, \eta_{\mathrm{p}}{ }^{2}=$ 0.26 . No such effect was found for target-same judgments ( $F$ $<1)$.

Thus, the results show that a change in the backdrop grouping produced a congruency effect on performance in the change-detection task on the target, suggesting that columns/ rows organization by proximity can be achieved without attention. Note that although different responses seem to be also less accurate when the backdrop changed, the significant congruency effect on target-different judgments was not due to a speed-accuracy trade-off, as indicated by the negative correlation between correct RT and accuracy in the target-different condition $(r=-.17)$.

The percentage of participants who responded correctly to the two surprise questions following the last experimental trial and the corresponding chi-square tests are presented in Table 1 . The percentage of participants who correctly reported the backdrop organization, as well as the percentage of participants who correctly reported whether or not the backdrop organization had changed on the preceding trial, were not significantly different from chance. These results confirmed that the backdrop organization was unattended.

\section{Experiment 2: Shape similarity grouping}

Data from three participants were excluded from the analysis of Experiment 2 due to performance at chance level in at least one of the conditions. As in Experiment 1, the analysis showed a significant main effect of backdrop organization on accuracy but not RT $(F<1)$, indicating that responses were

Table 1 Percentage of participants who responded correctly and results of corresponding chi-square tests, for each forced-choice question in Experiments 1 and 2

\begin{tabular}{lllll}
\hline Experiment & Question & Correct reports & Chi-square value & $p$ value \\
\hline Experiment 1 & Pattern & $60 \%(12 / 20)$ & 1.17 & .28 \\
& Change & $55 \%(11 / 20)$ & 0.2 & .65 \\
Experiment 2 & Pattern & $50 \%(14 / 28)$ & 0.1 & .75 \\
& Change & $57 \%(16 / 28)$ & 0.48 & .49 \\
& Shapes & $46 \%(13 / 28)$ & 0.19 & .66 \\
\hline
\end{tabular}

more accurate (by $2.6 \%$ ) when the organization in the backdrop stayed the same than when it changed: $\mathrm{AC}, F(1,27)=$ 9.04, $p<.007, \eta_{\mathrm{p}}^{2}=0.25$. A main effect of target was also found: Same responses were significantly more accurate (by $3.4 \%$ ) and tended to be faster (by $25 \mathrm{~ms}$ ) than different responses: AC, $F(1,27)=6.23, p<.02, \eta_{\mathrm{p}}{ }^{2}=0.19$; RT, $F(1,27)$ $=3.7, p=.07, \eta_{\mathrm{p}}{ }^{2}=0.12$. Importantly, unlike in Experiment 1 , the interaction between target and backdrop conditions was not significant for accuracy or RT: AC, $F(1,27)=1.84, p=$ $.19, \eta_{\mathrm{p}}{ }^{2}=0.06$; RT, $\left.F<1\right]$, indicating that the backdrop organization did not produce congruency effects on the changedetection task. This result suggests that grouping into columns/rows by shape similarity requires attention in order to be achieved.

As in Experiment 1, both the percentage of participants who correctly reported the backdrop organization and the percentage of participants who correctly reported whether or not the backdrop organization had changed were not significantly different from chance (see Table 1). In addition, the percentage of participants that correctly identified the local shapes was also not significantly different from chance. These results confirm that the backdrop was unattended.

To rule out the possibility that the lack of congruency effects in Experiment 2 was a result of low discriminability of the organizations, an additional experiment was conducted. In this experiment, 20 new participants were instructed to attend to the backdrop displays, which were presented exactly as in the inattention experiment. On each trial, the participants were requested to report the organization they perceived in the second display, and whether or not they noticed a change in the organization between consecutive displays. We hypothesized that the participants would be able to identify the organizations and to detect a change in the organization between displays when their attention was not engaged elsewhere. As expected, performance was very good: participants correctly reported whether the organization in the preceding display was columns or rows on $88 \%$ of the trials, and were accurate in reporting whether or not there was a change in the organization between displays on $85 \%$ of the trials. These results confirm that the organizations by shape similarity were easily perceived when they were attended.

The finding that same responses tended to be faster than different responses, which was observed also in Experiment 1, is reminiscent of the fast-same effect, which is commonly found in studies that use a same-different judgment task (Farell, 1985), in particular when the stimuli to be compared are not easily discriminable (Eriksen, 1995, p. 108), as in the current change-detection task of the central matrices.

The results of Experiments 1 and 2 show that changes in the unattended backdrop organization produced a congruency effect on performance in the change-detection task on the target matrix in Experiment 1 (grouping by proximity) but not in Experiment 2 (grouping by shape similarity). A three- 
way mixed-design ANOVA (experiment as a betweensubjects factor and target and backdrop organization as within-subjects factors) provides some support for these findings. The interaction between target, backdrop organization, and experiment was marginally significant for RT, $F(1$, $46)=3.23, p=.08, \eta_{\mathrm{p}}{ }^{2}=0.07$. This difference in congruency effect was observed despite the fact that responses to the surprise questions that followed the last trial confirmed that the backdrop was indeed unattended in both experiments, as participants were at chance level reporting the organization or a change in it (Experiments 1 and 2), or even the local shapes of the elements (Experiment 2). Also, the finding of no congruency effects in Experiment 2 could not be attributed to low discriminability of the organizations, as demonstrated by the additional experiment, where participants were highly accurate reporting the organization in the backdrop or a change in it when their attention was directed to the backdrop display. Thus, these results indicate that grouping into columns/rows by proximity can be accomplished under inattention, whereas there was no evidence for grouping into an equivalent organization by shape similarity.

As noted earlier, previous findings showed that grouping by proximity and grouping by shape similarity have different time courses, such that the latter requires more time than the former to be accomplished (e.g., Ben-Av \& Sagi, 1995; Han et el., 2001). For example, participants in Ben-Av and Sagi's (1995) study indicated the orientation of a masked display of columns/rows grouped by proximity or by shape similarity, while the SOA between the display and a backward mask was varied. Evidence of proximity grouping was found with SOA of $60 \mathrm{~ms}$, but shape similarity grouping was evident only with SOA of $160 \mathrm{~ms}$. Han et al. (2001) showed the same propensity with ERP recordings: grouping by proximity was indicated by a positive activity at $100-120 \mathrm{~ms}$ after stimulus presentation over the medial occipital cortex, and a negative activity with an onset of $180 \mathrm{~ms}$ over the occipitoparietal cortex, whereas grouping by shape similarity was reflected only in a longlatency occipitotemporal negativity with an onset of $260 \mathrm{~ms}$. The present results show that grouping by proximity and grouping by shape similarity differ not only in their time course but also in attentional demands: grouping by proximity appears to be accomplished without attention, whereas grouping by shape similarity appears to require attention.

The results of the current experiments provide evidence for a differential demand for attention as a function of the grouping principles involved. These results also demonstrate that attentional demands can be elicited when no competition for figural assignment is involved. The next three experiments were designed to further extend these conclusions by examining other processes that may elicit attentional demands in grouping.

\section{Experiments 3, 4, and 5}

Grouping organizations often involve shape formation more complex than the rows/columns organizations examined in the previous experiments, requiring the formation of a distinct shape rather than just determining the orientation of the segregated groups. The results of Experiment 2 suggest that attentional demands can arise when shape formation is relatively simple but element segregation is demanding. Another possible cause for attentional requirements in grouping may be the need to complete contingent processes of element segregation and shape formation in order to construct the organization of a distinctive shape. For example, Kimchi and Razpurker-Apfeld (2004) showed that grouping organizations that involve configuring elements into a shape, such as a cross or a square, could be accomplished under inattention. However, this was true only when a single homogeneous group of elements was present. When the display comprised of elements that varied in color, and segregation of elements was involved, the organization of a shape by color similarity was not obtained without attention. As mentioned in the introduction, Kimchi and Razpurker-Apfeld (2004) suggested that attention was required in this case in order to resolve figure-ground relations between the segregated groups. However, it is possible that the need for attention was caused not by the need to resolve figural assignment but rather by the need to accomplish the contingent processing of element segregation according to color similarity and configuring elements into a distinct shape, in order to form a coherent organized percept. Thus, Experiments 3, 4, and 5 examined whether the need for attention can arise due to such contingent processing. To this end, the backdrop organizations in these experiments were comprised of a shape configured from a subgroup of elements in the display (Experiments 3 and 5) or from a single group of elements (Experiment 4). In Experiments 3 and 5, to disentangle process complexity from competition over figural status, the segregated group that formed a shape comprised the most likely candidate for figural status, because the other elements in the display did not form any cohesive group. Thus, in Experiment 3, the organizations included a shape formed by collinear oval elements surrounded by randomly oriented elements (see Fig. 4a), and in Experiment 5 the organizations included a shape formed by connected elements, with additional nonconnected elements in the display (see Fig. 4c). Hence, the organizations in the backdrop involved both element segregation and shape formation, but the need to resolve figure-ground relations was markedly reduced. If such grouping can be accomplished without attention, then congruency effects, indicated by the interaction between target and backdrop conditions, are expected to emerge. However, if this complex processing cannot be accomplished without attention, then no congruency effects should emerge. For comparison, in Experiment 4 the 
A

Experiment 3 Segregation \&

Configuring by

Collinearity

\section{B}

\section{Experiment 4 Shape \\ Configuring}

\section{C}

Experiment 5 Segregation \& Configuring by

Element

Connectedness

D

Experiment 6

Segregation \&

Configuring by

Connectedness 1st Display
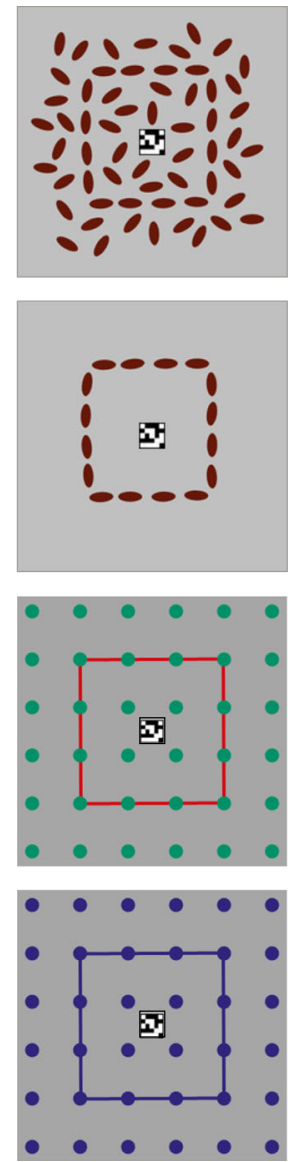

Fig. 4 Examples of the backdrop stimuli employed in (a) Experiment 3 (element segregation and shape formation by collinearity), (b) Experiment 4 (shape formation), (c) Experiment 5 (element segregation

backdrop organizations included only the collinear elements that formed a shape so that no element segregation was involved (see Fig. 4b). In line with previous findings demonstrating that such grouping can occur under inattention (Kimchi \& Razpurker-Apfeld, 2004), we expected congruency effects to emerge in this experiment.

Note that Experiment 5 involved the grouping principle of element connectedness. This is a modern grouping principle (Palmer \& Rock, 1994), which states that elements that are connected by other elements tend to be grouped together, and unlike most classical grouping principles that are intrinsicbased on some property of the elements themselves (e.g., color, location, shape) - it is an extrinsic grouping principle, in which grouping of several elements is based on their relations with contextual elements (e.g., Palmer \& Beck, 2007). It has been demonstrated that this principle can overcome similarity by shape, and even the effect of proximity (Brooks, 2015; Palmer \& Rock, 1994; Palmer, 1999). Thus, examining attentional demands for organizations involving the principle of

2nd Display

Same

Organization
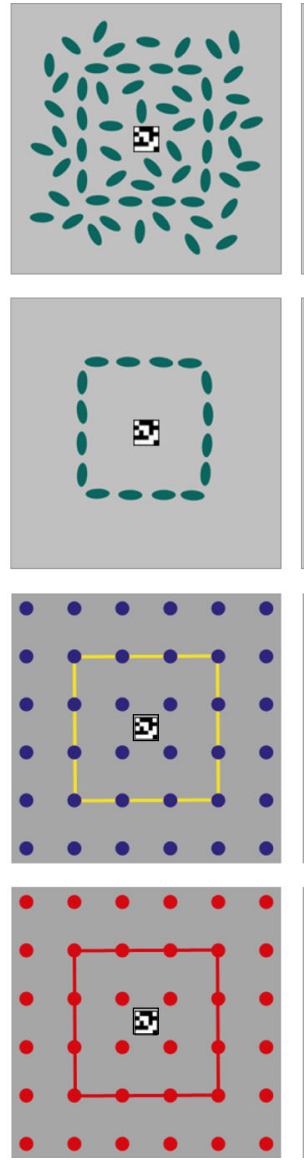

2nd Display

Different

Organization
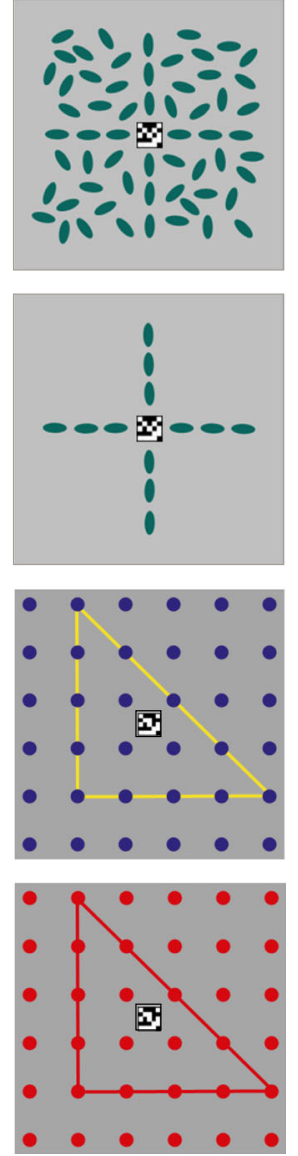

and shape formation by element connectedness), and (d) Experiment 6 (element segregation and shape formation by connectedness - uniform color). (Color figure online)

element connectedness would provide additional and desired information concerning this principle.

\section{Method}

\section{Participants}

Eighteen students from the University of Haifa participated in Experiment 3, another 18 participated in Experiment 4, and 20 others participated in Experiment 5. All the participants had normal or corrected-to-normal vision and normal color vision. None participated in more than one experiment, and none participated in the previous experiments.

Stimuli, apparatus, design, and procedure

The stimuli, apparatus, design, and procedure for Experiments 3,4 , and 5 were the same as in the Experiments 1 and 2, except for the following. 
Experiment 3: Element segregation and shape formation by collinearity The elements in the backdrop organization were solid ellipses $\left(1^{\circ} \times 0.5^{\circ}\right.$ each $)$ in various orientations. Each display consisted of 60 elements of one color (red or green), scattered on a $9.5^{\circ} \times 9.5^{\circ}$ area around the target matrix. A subset of the elements in each display was grouped by collinearity to form a cross (12 elements) or a square (16 elements). The other elements of each display were randomly oriented, and their density was approximately the same in all the displays (15 elements per quarter). The distance of the most central elements from the target matrix was $0.4^{\circ}$. The color and orientation of the elements always changed between successive displays (red in the first and green in the second, or vice versa), and some elements changed their location, regardless of whether or not the grouping changed, to control for the possibility of detecting a change in the backdrop grouping from local changes of just a few elements. Examples of the stimulus displays are presented in Fig. 4a.

Experiment 4: Shape formation In this experiment, the backdrop organizations included only one group of collinear elements forming a shape (i.e., a square or across). Examples of the stimulus displays are presented in Fig. $4 \mathrm{~b}$.

\section{Experiment 5: Element segregation and shape formation} by element connectedness Each backdrop display consisted of a $6 \times 6$ solid dots, each subtended $0.48^{\circ}$ in diameter, on a light-gray background. The distance between adjacent elements was $1.2^{\circ}$. The distance of the most central elements from the target matrix was $0.3^{\circ}$. A subset of 12 elements was connected by bars of a different color forming either a square or a triangle. To control for the possibility of detecting a change in the backdrop grouping from local changes in the elements, the dots and the connecting bars always changed their colors between successive displays (green dots and red bars in the first display and blue dots and yellow bars in the second, or vice versa). Examples of the stimulus displays are presented in Fig. 4c.

In all three experiments, after the last experimental trial, the participants were asked two forced-choice questions. The first question asked was, "What was the shape in the backdrop?" The two alternatives were cross or square in Experiments 3 and 4, and square or triangle in Experiment 5. The second question asked was, "Was there a change in the backdrop organization across the two displays in the previous trial?" The two alternatives were change and no change.

\section{Results and discussion}

Accuracy and correct RT data were subjected to a repeated measures two-way ANOVA (Target $\times$ Backdrop
Organization) for each experiment. RTs less than $250 \mathrm{~ms}$ and greater than $2,000 \mathrm{~ms}$ were discarded $(0.2 \%, 0.4 \%$, and $0.3 \%$ of all trials in Experiments 3, 4, and 5, respectively). Figure 5d depicts mean ACs and correct RTs for same and different targets as a function of backdrop organization (same, different) for Experiment 3 (Fig. 5a; element segregation and shape formation by collinearity), Experiment 4 (Fig. 5b; shape formation), and Experiment 5 (Fig. 5c; element segregation and shape formation by element connectedness).

\section{Experiment 3: Element segregation and shape formation} by collinearity

A significant main effect of backdrop organization was found on the accuracy data but not on RT, indicating that responses were more accurate (by $2.4 \%$ ) when the organization in the backdrop stayed the same than when it changed: AC, $F(1,17)=4.78, p<.05, \eta_{\mathrm{p}}{ }^{2}=0.22$; RT, $F<1$. No effect of target was found $(F \mathrm{~S}<1)$ for $\mathrm{AC}$ and RT. The interaction between target similarity and backdrop similarity was not significant: AC, $F<1$; RT, $F(1$, $17)=1.6, p=.22, \eta_{\mathrm{p}}{ }^{2}=0.09$, indicating that the backdrop organization did not produce congruency effects on the change-detection task (see Fig. 5a). Thus, there is no evidence to suggest that the backdrop organization was achieved under inattention.

The percentage of participants who responded correctly to the two surprise questions following the last experimental trial and the corresponding chi-square tests are presented in Table 2. The percentage of participants who correctly reported the backdrop organization, as well as the percentage of participants who correctly reported whether or not the backdrop organization had changed on the preceding trial, were not significantly different from chance, confirming that the backdrop organization was unattended.

To ensure that the lack of congruency effects in this experiment was not due to low discriminability of the backdrop organizations, an additional experiment was conducted, similar to the one conducted for Experiment 2 , in which participants attended to the backdrop organization. Fourteen new participants correctly reported whether the shape in the organization was a cross or a square on $94 \%$ of the trials, and whether or not there was a change in the backdrop organization between the first and second display on $91 \%$ of the trials. These results confirm that when attention was allocated to the organizations they were highly discriminable. Hence, the lack of congruency effects reported in Experiment 3 resulted from the lack of attention to the backdrop displays.

Thus, the results of this experiment suggest that grouping involving elements segregation and shape formation by 


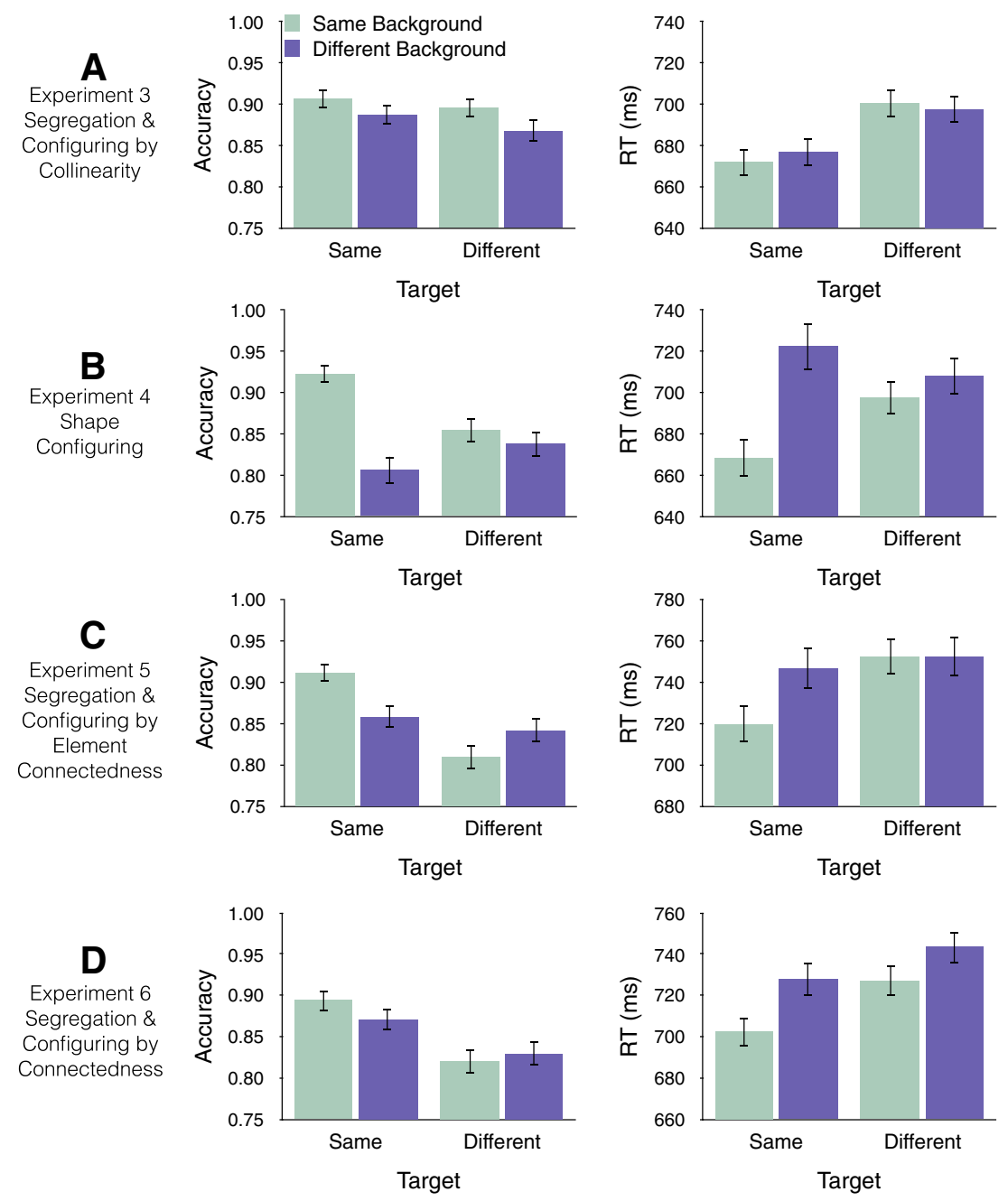

Fig. 5 Mean accuracy (left column) and correct RTs (right column) for same and different targets as a function of backdrop organization (same, different) in (a) Experiments 3 (element segregation and shape formation by collinearity), (b) Experiment 4 (shape formation), (c) Experiment 5

collinearity may not be accomplished under inattention, as no evidence of such grouping has been found.

Table 2 Percentage of participants who responded correctly and results of corresponding chi-square tests, for each forced-choice question in Experiments 3-6

\begin{tabular}{lllll}
\hline Experiment & Question & Correct reports & Chi-square value & $p$ value \\
\hline Experiment 3 & Shape & $39 \%(7 / 18)$ & 0.47 & 0.49 \\
& Change & $61 \%(11 / 18)$ & 0.75 & 0.39 \\
Experiment 4 & Shape & $47 \%(7 / 15)$ & 0.27 & 0.61 \\
& Change & $47 \%(7 / 15)$ & 1.88 & 0.17 \\
Experiment 55 & Shape & $34 \%(6 / 18)$ & 1.3 & 0.26 \\
& Change & $50 \%(9 / 18)$ & 0 & 1 \\
Experiment 6 & Shape & $56 \%(10 / 18)$ & 0.23 & 0.63 \\
& Change & $61 \%(11 / 18)$ & 0.9 & 0.24 \\
\hline
\end{tabular}

(element segregation and shape formation by element connectedness), and (d) Experiment 6 (element segregation and shape formation by connectedness). Error bars indicate standard errors of the means. (Color figure online)

\section{Experiment 4: Shape formation}

Data from three participants were excluded from the analysis due to performance at chance level in at least one of the conditions. A significant main effect of backdrop organization was found on the accuracy and RT data, indicating that responses were more accurate (by 6.7\%), and faster (by $33 \mathrm{~ms}$ ), when the organization in the backdrop stayed the same than when it changed: AC, $F(1,14)=15.58, p<.003, \eta_{\mathrm{p}}{ }^{2}=0.53$; RT, $F(1,14)=14.74, p<.003, \eta_{\mathrm{p}}{ }^{2}=0.51$. No effect of target was found $\left(F_{\mathbf{S}}<1\right)$. Importantly, as predicted, a significant interaction between target and backdrop conditions was found on both accuracy and RT: AC, $F(1,14)=9.08, p<.01, \eta_{\mathrm{p}}{ }^{2}=$ 0.39; RT, $F(1,14)=7.72, p<.02, \eta_{\mathrm{p}}{ }^{2}=0.36$ (see Fig. $5 \mathrm{~b}$ ). Planned comparisons confirmed congruency effects for the same-target condition: target-same judgments were more accurate (by $11.67 \%$ ) and faster (by $54 \mathrm{~ms}$ ) when the backdrop stayed the same than when it changed: AC, $F(1,14)=17.77, p$ 
$<.001, \eta_{\mathrm{p}}{ }^{2}=0.56$; RT, $F(1,14)=18.7, p<.001, \eta_{\mathrm{p}}{ }^{2}=0.57$. No such effect was found for the different-target condition: AC, $F<1$; RT, $F(1,14)=1.5, p>.24, \eta_{\mathrm{p}}{ }^{2}=0.1$. Thus, congruency effects produced by changes in the backdrop organization on performance in the change-detection task were found, even though the responses to the two surprise questions following the last experimental trial (see Table 2) showed that participants could not correctly report the backdrop organization or whether the organization changed between consecutive displays. These results suggest that configuring the collinear elements into a shape with no need for elements segregation could be accomplished under inattention.

\section{Experiment 5: Element segregation and shape formation by element connectedness}

Data from two participants were excluded from the analysis due to performance at chance level in at least one of the conditions. A significant main effect of target was found with both accuracy and RT, indicating that responses were more accurate (by $5.89 \%$ ), and faster (by $20 \mathrm{~ms}$ ), when the target stayed the same than when it changed: $\mathrm{AC}, F(1,17)=4.41, p=.05, \eta_{\mathrm{p}}{ }^{2}=$ 0.21 ; RT, $F(1,17)=4.55, p<.05, \eta_{\mathrm{p}}{ }^{2}=0.21$. The main effect of backdrop organization did not reach significance: AC, $F<$ 1 ; RT, $F(1,17)=2.9, p=.11, \eta_{\mathrm{p}}{ }^{2}=0.15$. Interestingly, the interaction between target and backdrop organization was significant for accuracy and marginally significant for RT: AC, $F(1,17)=4.96, p<.04, \eta_{\mathrm{p}}{ }^{2}=0.23$; RT, $F(1,17)=3.56, p=$ $.08, \eta_{\mathrm{p}}{ }^{2}=0.17$. Planned comparisons confirmed congruency effects for the same-target condition: target-same judgments were more accurate (by 5.29\%) and faster (by $27 \mathrm{~ms}$ ) when the backdrop stayed the same than when it changed: AC, $F(1,17)$ $=5.29, p<.04, \eta_{\mathrm{p}}{ }^{2}=0.24$; RT, $F(1,17)=4.85, p<.05, \eta_{\mathrm{p}}{ }^{2}=$ 0.22 . No such effect was found for the different-target condition: $\mathrm{AC}, F(1,17)=2.02, p=.17, \eta_{\mathrm{p}}{ }^{2}=0.11$; RT, $F<1$. Thus, the results show that a change in the backdrop organization produced congruency effects on performance in the changedetection task. The congruency effects were obtained even though the participants were at chance level reporting the organization in the backdrop or a change in it between displays (see Table 2). Thus, the results of this experiment suggest that an organization that involves both element segregation and shape formation can be accomplished under inattention when based on element connectedness.

The goal of Experiments 3, 4, and 5 was to examine whether contingent processing of element segregation and shape formation into distinct shapes require attention. The results showed that when the backdrop organization involved only shape formation of one group of elements (Experiment 4), changes in the unattended backdrop organization produced congruency effects on performance in the change-detection task on the target matrix, replicating results from a similar condition in Kimchi and Razpurker-Apfeld (2004). These results indicate that when configuring elements into a shape is not contingent on elements segregation the organization can be accomplished under inattention. However, no congruency effects were observed in Experiment 3, in which backdrop organizations involved both elements segregation by collinearity and shape formation. This lack of evidence of background organization suggests that this organization may not be accomplished under inattention. Interestingly, however, congruency effects, indicating backdrop organization under inattention, were found in Experiment 5, in which the organization also involved both element segregation and shape formation but was presumably governed by the grouping principle of element connectedness. A three-way mixed-design ANOVA (experiment as a between-subjects factor and target and backdrop organization as within-subjects factors) conducted on the data of Experiments 3 and 5 supports the difference in congruency effects between the two experiments: the interaction between target, backdrop organization and experiment was significant for accuracy, $F(1,31)=7.44, p<.02, \eta_{\mathrm{p}}{ }^{2}$ $=0.19$.

This difference in congruency effects between Experiments 3 and 5 is not likely to be due to different degrees of inattention, or to low discriminability of the organizations in Experiment 3. Surprise questions following the last experimental trial confirmed that the backdrop was indeed unattended in all the experiments, as participants were at chance level reporting the organization in the preceding display or whether a change had occurred in the organization between the last two displays. Also, an additional experiment confirmed that the results of Experiment 3 could not be explained by low discriminability of the organizations, as participants were highly accurate reporting the organizations employed in this experiment and a change in them when attending the organizations. Thus, the results of Experiments 3 suggest that contingent processing of element segregation and shape formation can raise the need for attention, even when there is almost no need to resolve figure-ground relations. On the face of it, the results of Experiment 5 demonstrate that grouping involving both element segregation and shape formation can be accomplished under inattention when the underlying grouping principle is element connectedness. However, an inspection of the displays used in this experiment (see Fig. 4c) suggests that when introducing element connectedness, we inadvertently introduced additional grouping principle of color similarity, which may be involved in the segregation and configuring, in addition to element connectedness. Previous findings showed that contingent processing of element segregation and shape formation by color similarity cannot be accomplished without attention (see Kimchi \& Razpurker-Apfeld, 2004). However, it is possible that the combination of the grouping principles of element connectedness and color similarity alleviate the attentional demands of the organization, thus accounting for the results of Experiment 5. To examine this account, we 
conducted Experiment 6, in which the connecting lines were the same color as the dots in the display, so that only connectedness was involved in the organization.

\section{Experiment 6}

Experiment 6 aimed to further examine the need for attention for organizations by connectedness when contingent processes of shape formation and segregation are required. The backdrop displays depicted similar organizations to those employed in Experiment 5, only all the elements in a display had the same color. This allowed us to test to what extent the effects observed in Experiment 5 were due to the connectedness per se or rather to the combination of connectedness and color similarity. That is, if connectedness can reduce attentional demands elicited by contingent processes, as the results of Experiment 5 may suggest, then congruency effects are expected to emerge for the organizations in Experiment 6 as well. However, if connectedness by itself is not enough, and the results of Experiment 5 are due to the combination of connectedness and color similarity, then no congruency effects are expected to be found.

\section{Method}

\section{Participants}

Twenty students from the University of Haifa participated in Experiment 6. All had normal or corrected-to-normal vision and normal color vision. None participated in the previous experiments.

\section{Stimuli, apparatus, design, and procedure}

The stimuli, apparatus, design, and procedure for Experiment 6 were the same as in Experiment 5, except that in each display the connecting lines were the same color as the dots (see Fig. 4d).

\section{Results and discussion}

Accuracy and correct RT data were subjected to a repeated measures two-way ANOVA (Target $\times$ Backdrop Organization). RTs less than $250 \mathrm{~ms}$ and greater than $2,000 \mathrm{~ms}$ were discarded ( $0.3 \%$ of all trials). Figure $5 \mathrm{~d}$ depicts mean ACs and correct RTs for same and different targets as a function of backdrop organization (same, different). Data from two participants were excluded from the analysis: one due to performance at chance level in at least one of the conditions and one due to extremely long reaction times (her mean RT was 2.9 SDs above the group mean). A significant main effect of target was found with accuracy but not RT, indicating that responses were more accurate (by 5.7\%) when the target stayed the same than when it changed: AC, $F(1,17)$ $=7.97, p<.02, \eta_{\mathrm{p}}{ }^{2}=0.32$; RT, $F(1,17)=2.32, p>.15, \eta_{\mathrm{p}}{ }^{2}=$ 0.12 . The main effect of backdrop organization was significant with RT indicating that responses were faster (by $20 \mathrm{~ms}$ ) when the backdrop stayed the same than when it changed: AC, $F<1$; RT, $F(1,17)=11.81, p<.004, \eta_{\mathrm{p}}^{2}=0.41$. Importantly, the interaction between target and backdrop organization did not reach significance for accuracy or for RT: AC, $F(1,17)=2.07, p>.17, \eta_{\mathrm{p}}{ }^{2}=.11$; RT, $F(1,17)=1.85, p$ $>.19, \eta_{\mathrm{p}}{ }^{2}=0.1$, indicating that the backdrop organization did not produce congruency effects on the change detection task (see Fig. 5d). As in the previous experiments, both the percentage of participants who correctly reported the backdrop organization, and the percentage of participants who correctly reported whether or not the backdrop organization had changed, were not significantly different from chance (see Table 2).

Thus, the results of this experiment show no evidence that an organization that involves both element segregation and shape formation based on connectedness can be accomplished under inattention. These results also suggest that the results of Experiment 5, indicating organization under inattention, were likely due to the combination of element connectedness and color similarity.

\section{General discussion}

This study aimed to further investigate the claim raised by Kimchi and Razpurker-Apfeld (2004) that attentional requirements vary for different perceptual grouping processes. In particular, two hypotheses were tested: (a) different grouping principles vary in their demand for attention and (b) the need for attention can arise when the grouping organization involves both element segregation and shape formation. These hypotheses were tested using the inattention paradigm (e.g., Driver et al., 2001; Kimchi \& Razpurker-Apfeld, 2004; Russell \& Driver, 2005), in which participants performed a demanding change-detection task on a small matrix presented on a task-irrelevant backdrop of grouped elements. Congruency effects produced by changes in the backdrop grouping on target-change judgments indicate grouping under inattention. The results provide support for the two hypotheses, but the picture that emerges concerning the attentional demands of perceptual grouping is far more intricate (see Table 3 for a summary of the results).

As predicted, different grouping principles showed different attentional demands: Congruency effects were observed when the backdrop elements grouped into columns/rows by proximity (Experiment 1) but not when they were grouped into columns/rows by shape similarity (Experiment 2), suggesting that the former could be accomplished without 
Table 3 Summary of the results in Experiments 1-6

\begin{tabular}{llll}
\hline Experiment & Grouping organization & $\begin{array}{l}\text { Congruency } \\
\text { effects }\end{array}$ & Attentional demands \\
\hline 1 & Rows/columns by proximity & Yes & No attention required \\
2 & Rows/columns by shape similarity & No & Attention required \\
3 & Element segregation and shape formation by collinearity & No & Attention required \\
4 & Shape formation with no element segregation due to collinearity & Yes & No attention required \\
5 & Element segregation and shape formation by connectedness (connecting lines differ in & Yes & No attention required \\
6 & color from connected dots) & No & Atttention required \\
& slement segregation and shape formation by connectedness (connecting lines have the & & \\
\hline
\end{tabular}

attention, whereas the latter requires attention. The finding regarding grouping by shape similarity is of particular interest in light of the previous finding that grouping into columns/ rows by color similarity can occur under inattention (Kimchi \& Razpurker-Apfeld, 2004; Russell \& Driver, 2005). Taken together, these findings suggest that not only different grouping principles can vary in their attentional demands but also that the grouping principle of similarity should not be treated as a single, unified principle, because attentional demands appear to depend on the type of similarity involved (e.g., similarity in color or similarity in shape).

The results further show that grouping that involves element segregation and shape formation can elicit attentional requirements. Congruency effects were observed when grouping into a square/cross did not involve element segregation (Experiment 4), suggesting that it was achieved without attention. However, no congruency effects were found when grouping into a distinct shape involved element segregation by collinearity (Experiment 3 ), or by connectedness (Experiment 6). No congruency effects were found also when shape formation was relatively simple but element segregation was more demanding, as in forming columns/rows by shape similarity (Experiment 2). Taken together, these results demonstrate that attentional demands can surface when grouping involves both elements segregation and shape formation (of different complexity), but attention is not required for grouping into a shape that does not involve element segregation, indicating that attentional demands depend on the complexity of the processes involved in the organization. However, the involvement of both element segregation and shape formation in grouping is clearly not sufficient for imposing attentional demands: organizations involving these processes were accomplished under inattention, both when shape formation and element segregation were relatively simple, as in grouping elements into columns/rows by proximity (Experiment 1), and when shape formation was more complex, forming a distinctive shape, as in grouping elements into square/triangle, supported by the combination of element connectedness and color similarity (Experiment 5). The latter is quite interesting, because it shows that although organization based on each of these grouping alone appeared to require attention (Experiment 6; Kimchi \& Razpurker-Apfeld 2004), the combination of the two principles seem to reduce attentional demands elicited by contingent processes.

The present findings also demonstrate that attentional demands can surface when there is no (or hardly no) need to resolve figure-ground relations. Thus, grouping into columns/ rows by shape similarity required attention (Experiment 2), although there was presumably no competition for figural status between the segregated columns or rows. Similarly, the results of Experiments 3 and 6 also show that grouping into a shape (square/cross/triangle) by collinearity or connectedness elicited attentional demands despite the fact that there was almost no competition for figural assignment, because only one group of segregated elements was the most likely candidate for figural status. These findings clearly suggest that the need to resolve figure-ground relations is not necessary for imposing attentional demands. Whether this need by itself suffices to impose attentional demands, as suggested by Kimchi and Razpurker-Apfeld (2004), is yet to be determined. The present results cannot rule out the possibility that the need to resolve a competition for figural assignment, which was present in the backdrop organizations employed by Kimchi and RazpurkerApfeld, contributed to the grouping's requirement for attention observed in their study.

Taken together, the results of the present study demonstrate that contingent processing of element segregation and shape formation, but not the need to resolve figure-ground relations, can elicit attentional demands. Yet whether or not attentional demands would surface depends on the particular grouping principles and the complexity of the processes involved in the organization. Thus, for example, grouping by shape similarity appears to require attention, even when simple shape formation is involved (Experiment 2), but grouping by proximity under the same conditions is accomplished under inattention. Yet when shape formation is more complex, forming a distinct shape, and element segregation is involved as well, attention appears to be required regardless of the simplicity of 
the grouping principle (Experiment 6), unless two grouping principles are combined (Experiment 5 ). These results support the suggestion that attentional demands vary as a function of the processes involved in the grouping organization and the circumstances in which each process takes place (Kimchi, 2009; Kimchi \& Razpurker-Apfeld, 2004). In addition, these results provide further evidence for the view that perceptual organization is a multiplicity of processes showing different attentional demands, time courses, and developmental progression (Behrmann \& Kimchi, 2003; Kimchi et al., 2005).

Any attempt to examine processing without attention comes with the concern of whether or not inattention conditions were indeed achieved (see also Kimchi \& RazpurkerApfeld, 2004). Yet the central task was extremely difficult and sufficiently demanding to absorb attention, and the backdrop organization was completely task irrelevant, so there was no incentive for the participants to deliberately allocate attention to it. A spillover of attention from the target to the backdrop should have two effects: First, it would likely reduce the ability to perform the central task and hence decrease performance, and, second, it would increase the ability to detect the backdrop organization and changes in it. The fact that overall performance level in the central task was maintained in all the experiments reported here, regardless of whether or not congruency effects were found, and that participants were at chance level answering the surprise questions about the background organizations in all the experiments, indicates that attention was allocated to the central task and the backdrop organizations were unattended. Moreover, attentional spillover suggests an effect of the distance between the target and the central backdrop elements, but the results show that congruency effects (or the lack of these effects) were independent of such distance. For example, the most central elements in Experiment 2 were closer to the target than those in Experiments 1, 4, and 5, but no congruency effects were observed in the former case and several were obtained in the latter. This possibility was also addressed and rejected by Russell and Driver (2005), who found congruency effects even when the elements most adjacent to the central matrix never changed their organization.

Finally, an effect of backdrop condition was found in all the experiments, showing worse performance when the backdrop organization changed compared with when it did not. The fact that this effect was found without an interaction between backdrop and target conditions is troubling, suggesting that a change in the backdrop was detected and impaired performance. This effect cannot be explained by the detection of local changes when the backdrop organization changed because local changes were present also when the organization did not change. It also cannot be explained by detection of global changes in the display, since no such global changes were present except for in the displays of Experiment 4 where an interaction emerged. The only systematic change was in the organizations depicted in the display. Thus, we suggest that this effect indicates some processing of the organization under inattention, which did not result in a strong representation that could lead to congruency effects.

Along this line, an anonymous reviewer suggested that the pattern of results demonstrated here could be accounted for by grouping strength/saliency. According to this account, no need for attention in grouping was observed (i.e., the emergence of congruency effects) when the organization was salient enough to produce a strong representation that could interact with the main task. Consequently, evidence of the involvement of attention (i.e., no congruency effects) was obtained when the organization was simply not salient enough. However, evaluating this account requires further investigation, since it is not clear what makes one organization more salient than another. For example, the shapes in Experiments 5 and 6 are very salient, but congruency effects were found in the former and not the latter. In addition, in Experiments 2 and 3, where no congruency effects were found, we conducted additional experiments where participants explicitly indicated the organization they perceived. Accuracy was very high in these experiments, indicating that the organizations were salient enough to be correctly identified once attention was not directed away from the backdrop. Thus, in order to examine the saliency account, a different kind of experimental design and measure should be devised.

In conclusion, this study provides a detailed demonstration of different circumstances that require attention in order for grouping to be achieved. Contingent processing of element segregation and shape formation appears to be necessary, but not sufficient, for imposing attentional demands. Attentional demands depend on the combination of grouping principles and the complexity of the processes involved in the grouping. Hence, although there is evidence of some organizations that do not require attention, attention might be necessary for many situations in perceptual organization.

Acknowledgements This research was supported by the Israel Foundation Trustees (IFT) Research Grant for Doctorates in Social Sciences to E.R. and by Max Wertheimer Minerva Center for Cognitive Processes and Human Performance, University of Haifa. The authors thank Anna Trostianitser and Irina Fridburg for their help in data collection.

\section{References}

Behrmann, M., \& Kimchi, R. (2003). What does visual agnosia tell us about perceptual organization and its relationship to object perception? Journal of Experimental Psychology: Human Perception and Performance, 29(1), 19-42.

Ben-Av, M. B., \& Sagi, D. (1995). Perceptual grouping by similarity and proximity: Experimental results can be predicted by intensity autocorrelations. Vision Research, 35(6), 853-866. 
Brooks, J. L. (2015). Traditional and new principles of perceptual grouping. In J. Wagemans (Ed.), Oxford handbook of perceptual organization (pp. 57-87). Oxford: Oxford University Press.

Ben-Av, M. B., Sagi, D., \& Braun, J. (1992). Visual attention and perceptual grouping. Perception \& Psychophysics, 52(3), 277-294.

Driver, J., Davis, G., Russell, C., Turatto, M., \& Freeman, E. (2001). Segmentation, attention and phenomenal visual objects. Cognition, 80(1/2), 61-95.

Eriksen, C. W. (1995). The flankers task and response competition: A useful tool for investigating a variety of cognitive problems. Visual Cognition, 2(2/3), 101-118.

Farell, B. (1985). "Same"-"different" judgments: A review of current controversies in perceptual comparisons. Psychological Bulletin, 98(3), 419-456. doi:10.1037/00332909.98.3.419

Hadad, B. S., \& Kimchi, R. (2006). Developmental trends in utilizing perceptual closure for grouping of shape: Effects of spatial proximity and collinearity. Perception \& Psychophysics, 68(8), 1264-1273.

Han, S. H. (2004). Interactions between proximity and similarity grouping: An event-related brain potential study in humans. Neuroscience Letters, 367(1), 40-43.

Han, S., Song, Y., Ding, Y., Yund, E. W., \& Woods, D. L. (2001). Neural substrates for visual perceptual grouping in humans. Psychophysiology, 38, 926-935.

Julesz, B. (1981). Textons, the elements of texture perception and their interactions. Nature, 290, 91-97.

Kimchi, R. (1998). Uniform connectedness and grouping in the perceptual organization of hierarchical patterns. Journal of Experimental Psychology: Human Perception \& Performance, 24, 1105-1118.

Kimchi, R. (2000). The perceptual organization of visual objects: A microgenetic analysis. Vision Research, 40, 1333-1347.

Kimchi, R. (2003). Visual perceptual organization: A microgenetic analysis. In R. Kimchi, M. Behrmann, \& C. R. Olson (Eds.), Perceptual organization in vision: Behavioral and neural perspectives (pp. 117-154). Mahwah: Erlbaum.

Kimchi, R. (2009). Perceptual organization and visual attention. Progress in Brain Research, 176, 15-33.

Kimchi, R., Hadad, B., Behrmann, M., \& Palmer, S. E. (2005). Microgenesis and ontogenesis of perceptual organization: Evidence from global and local processing of hierarchical patterns. Psychological Science, 16(4), 282-290.

Kimchi, R., \& Peterson, M. A. (2008). Figure-ground segmentation can occur without attention. Psychological Science, 19(7), 660-668.

Kimchi, R., \& Razpurker-Apfeld, I. (2004). Perceptual grouping and attention: Not all groupings are equal. Psychonomic Bulletin and Review, 11(4), 687-696.
Koffka, K. (1935). Principles of Gestalt psychology. London: Lund Humphries.

Kurylo, D. D. (1997). Time course of perceptual grouping. Perception \& Psychophysics, 59(1), 142-147.

Lamy, D., Segal, H., \& Ruderman, L. (2006). Grouping does not require attention. Perception \& Psychophysics, 68(1), 17-31.

Mack, A., Tang, B., Tuma, R., Kahn, S., \& Rock, I. (1992). Perceptual organization and attention. Cognitive Psychology, 24, 475-501.

Marr, D. (1982). Vision: A computational investigation into the human representation and processing of visual information. San Francisco: Freeman.

Moore, C., \& Egeth, H. (1997). Perception without attention: Evidence of grouping under conditions of inattention. Journal of Experimental Psychology: Human Perception and Performance, 23(2), 339-352.

Neisser, U. (1967). Cognitive psychology. New York: Appleton-CenturyCrofts.

Palmer, S. E. (1999). Vision science: Photons to phenomenology. Cambridge: MIT Press.

Palmer, S. E., \& Beck, D. M. (2007). The repetition discrimination task: An objective method for studying perceptual grouping. Perception \& Psychophysics, 69(1), 68-78.

Palmer, S. E., Brooks, J. L., \& Nelson, R. (2003). When does grouping happen. Acta Psychologica, 114, 311-330.

Palmer, S., \& Rock, I. (1994). Rethinking perceptual organization: The role of uniform connectedness. Psychonomic Bulletin and Review, l(1), 29-55.

Quinn, P. C., Bhatt, R. S., Brush, D., Grimes, A., \& Sharpnack, H. (2002). Development of form similarity as a Gestalt grouping principle in infancy. Psychological Science, 13(4), 320-328.

Rock, I. (1986). The description and analysis of object and event perception. In K. R. Boff, L. Kaufman, \& J. P. Thomas (Eds.), Handbook of perception and human performance (Vol. 2, pp. 33.1-33.71). New York: Wiley.

Rock, I., Linnett, C. M., Grant, P., \& Mack, A. (1992). Perception without attention: Results of a new method. Cognitive Psychology, 24(4), 502-534.

Russell, C., \& Driver, J. (2005). New indirect measures of "inattentive" visual grouping in a change-detection task. Perception \& Psychophysics, 67(4), 606-623.

Treisman, A. (1982). Perceptual grouping and attention in visual search for features and for objects. Journal of Experimental Psychology: Human Perception \& Performance, 8, 194-214.

Trick, L. M., \& Enns, J. T. (1997). Clusters precede shapes in perceptual organization. Psychological Science, 8, 124-129. 